1

2

3

\title{
Improvement of Vickers hardness measurement on $\mathrm{SWNT} / \mathrm{Al}_{2} \mathrm{O}_{3}$ composites consolidated by spark plasma sintering
}

\author{
A. Morales-Rodríguez ${ }^{\mathrm{a}, \mathrm{b}^{*}}$, A. Gallardo-López ${ }^{\mathrm{a}, \mathrm{b}}$, A. Fernández-Serrano ${ }^{\mathrm{a}}$, \\ R. Poyato ${ }^{\mathrm{b}}, \mathrm{A}$. Muñoz ${ }^{\mathrm{a}}$ and A. Domínguez-Rodríguez ${ }^{\mathrm{a}}$ \\ ${ }^{a}$ Department of Condensed Matter Physics, Universidad de Sevilla, P.O. BOX 1065, \\ 41080 Sevilla, Spain \\ ${ }^{\mathrm{b}}$ Materials Science Institute of Sevilla (CSIC-Universidad de Sevilla), Américo \\ Vespucio 49, 41092 Sevilla, Spain \\ *Corresponding author. Tel.: +34 954556028; fax: +34 954552870; e-mail: amr@us.es
}

\begin{abstract}
Dense alumina composites with different carbon nanotube content were prepared by colloidal processing and consolidated by Spark Plasma Sintering (SPS). Single-wall carbon nanotubes (SWNTs) were distributed at grain boundaries and also into agglomerates homogeneously dispersed. Carrying out Vickers hardness tests on the cross-section surfaces instead of top (or bottom) surfaces has shown a noticeable increase in the reliability of the hardness measurements. This improvement has been mainly attributed to the different morphology of carbon nanotube agglomerates, which however does not seem to affect the Vickers hardness value. Composites with lower SWNT content maintain the Vickers hardness of monolithic alumina, whereas it significantly decreases for the rest of compositions. The decreasing trend with increasing SWNT content has been explained by the presence of higher SWNT quantities at grain boundaries. Based on the results obtained, a method for optimizing Vickers hardness tests performance on $\mathrm{SWNT} / \mathrm{Al}_{2} \mathrm{O}_{3}$ composites sintered by SPS is proposed.
\end{abstract}

Keywords: Carbon nanotubes; Alumina; Nanocomposites; Spark plasma sintering; Vickers hardness 


\section{Introduction}

Based on the exceptional combination of axial strength and resilience of high-aspectratio carbon nanotubes (CNTs) [1-4], either single-wall or multi-wall (MWNTs), the development of composites has been significantly promoted in the last decade. Much of the work has been focused on $\mathrm{CNT} / \mathrm{Al}_{2} \mathrm{O}_{3}$ composites, specially using MWNTs despite their "sword and sheath" failure type make them less structurally stable than SWNTs. A great variety of conflicting results are reported in literature. In some works no reinforcement is observed [5-7] while in others fracture toughness $\left(\mathrm{K}_{\mathrm{IC}}\right)$ increases [816], but no clear trend is found when changing the amount of CNTs.

The effect of CNT addition on composite hardness is not well established either. While several studies found the composite hardness worsens noticeably incorporating carbon nanotubes compared to the hardness value of monolithic alumina [7,8,10,13-15], other authors reported enhanced hardness for composites with CNTs [11,12,16,17]. For instance, $\mathrm{Yi}$ et al [10] found a decreasing trend in Vickers hardness of SWNT/ $\mathrm{Al}_{2} \mathrm{O}_{3}$ composites prepared by combustion reaction and quick pressing. By adding 0.5, 1 and 2 wt. \% SWNT, hardness values dropped $21 \%, 26 \%$ and 58\% respectively as compared with alumina. Bakhsh et al [14] also found decreasing hardness values for 1, 2 and $3 \mathrm{wt}$. $\% \mathrm{MWNT} / \mathrm{Al}_{2} \mathrm{O}_{3}$ composites prepared by conventional sintering in flowing argon atmosphere. On the contrary, Mo et al [11] found that MWNT addition of 1.5 and 3 vol. $\%$ slightly enhanced the hardness of CNT/alumina composites with an increase of 5\% and $7 \%$ respectively as compared with monolithic alumina.

Regarding top views of Vickers indentation sites published elsewhere [6,10], it should be noted that the diagonals of indenter imprint are not observed in the composite with carbon nanotubes. Recently, Thomson et al. [7] have questioned the validity of the Vickers method to quantify the hardness in 10 vol. \% SWNT/ $\mathrm{Al}_{2} \mathrm{O}_{3}$ composites fabricated by Wang et al. [6] because the surface finishing was not acceptable, arguing that the presence of large pores impedes indents measuring. However, no considerations about the absence of impression of the pyramid's edges after Vickers indentation were discussed. 
Heterogeneous dispersion and distribution of CNTs in the ceramic matrix, poor chemical compatibility between CNTs and alumina hindering effective transfer load and the large differences in the scales of the matrix microstructure and the carbon nanotubes have been stated as main obstacles to transfer the desirable CNT mechanical properties to the brittle ceramic matrix [5,7,11,14,15]. Adequate dispersion of CNTs is very difficult owing to their tendency to form bundles in order to minimize their surface area. Although aqueous colloidal processing has been assessed as an efficient technique producing adequate dispersion of CNTs throughout ceramic matrix grain boundaries after sintering [18-20], the presence of agglomerates seems to be unavoidable. Recently, Poorteman et al [21] fabricated MWNT/alumina composites with low MWNT content $(0.6$ and 1.4 vol. \%) by a colloidal processing route to optimize electrostatic repulsion. The suspension was rapidly frozen with liquid nitrogen followed by freezedrying to preserve the homogeneity of the mixture. Even those hot-pressed composites showed extended CNT agglomerated zones ( $50 \mu \mathrm{m}$ size) despite all the precautions taken.

The presence of agglomerated CNTs, usually related to high CNT concentration, has been pointed out as responsible for both the decrease of the fracture toughness $[11,14,15]$ and for the same decreasing trend followed by the hardness values with increasing CNT content [14]. Moreover, sintering of CNT/alumina composites usually results in reduced density with increasing amount of CNTs in the matrix [14,16]. This reduction in density has been attributed to the presence of agglomerates and leads to a reduction in mechanical properties. Recently, Sarkar and Das [16] incorporated MWNTs into $\mathrm{Al}_{2} \mathrm{O}_{3}$ from a low level (i.e. 0.15 vol. \%) to minimize the effect of severe agglomeration. These authors found a trend of decreasing sinterability with increasing CNT content above 0.6 vol. \% MWNT and pointed out that with only 1.2 vol. \% MWNT, the aggregates acted as pores of similar dimensions playing a negative role in densification. It is assumed that agglomeration becomes relevant for high CNT content due to dispersion difficulties during processing [18], however no systematic studies have been carried out to quantify this assumption. Unfortunately, this lack of publications providing quantitative information to characterize the agglomeration of CNTs impedes to precisely compare the goodness among different processing methods and dispersion routines. 
Besides the CNT tendency to gather and the difficulty above mentioned for comparison, the mechanical properties of brittle ceramic matrix composites present an inherent substantial scatter. Large scatter of mechanical data-set should be analyzed according to statistical methods for component designing with these materials. Promising results in this direction have been recently published [22]. This study revealed that MWNTs enhance mechanical properties of $\mathrm{MWNT} / \mathrm{Al}_{2} \mathrm{O}_{3}$ compared to pure alumina and the data treatment performed with statistical tools suggested better structural reliability of the former [22]. Unfortunately, reliability studies of mechanical properties of CNT/ceramic composites are extremely limited, which keeps away the actual real-life application of these composites.

In this paper, aqueous colloidal processing of SWNTs and alumina powder has been performed to minimize the heterogeneity in the CNT dispersion and composite powders were subsequently consolidated by SPS to avoid damage the carbon nanotubes and excessive grain growth of the matrix. The SWNT agglomerates distribution has been characterized for the different SWNT content and the Vickers hardness has been evaluated for these $\mathrm{SWNT} / \mathrm{Al}_{2} \mathrm{O}_{3}$ composites. Additionally, the effect of agglomerate distribution on the Vickers imprint formation has been addressed for the first time. Weibull statistical analysis of Vickers hardness data has also been performed to assess the reliability of $\mathrm{SWNT} / \mathrm{Al}_{2} \mathrm{O}_{3}$ composites for structural applications and to establish the actual effect of the agglomerates on mechanical properties of CNT/alumina composites. In view of the results obtained, a method for improving hardness measurements in such composites has been proposed.

\section{Experimental procedure}

\subsection{Raw materials and processing}

Monolithic polycrystalline alumina and SWNT/alumina composites with different carbon nanotube content $(1,3,5$ and 10 vol. \% SWNT) were prepared from $\alpha$-alumina powder (99\% purity and 30-40 nm particle size) supplied by Nanostructured and Amorphous Materials Inc. (Houston, TX) and HiP-co SWNTs provided by Carbon Solutions Inc. (Riverside, CA). Details about colloidal processing of composite powder with acid treated SWNTs have been reported elsewhere [19]. This procedure was 
specifically developed to achieve a highly homogeneous distribution of SWNTs at ceramic grain boundaries [19].

The materials were consolidated by SPS (Syntex Inc. Model 515S, Dr Sinter Inc., Kanagawa, Japan) using the following sintering conditions: sintering temperature of $1300^{\circ} \mathrm{C}$ for 5 minutes, applied uniaxial pressure of $75 \mathrm{MPa}$, and heating and cooling ramps of 300 and $50{ }^{\circ} \mathrm{C} / \mathrm{min}$, respectively. These conditions were selected based on a previous study of the authors [23] devoted to optimize the sintering conditions for monolithic alumina in order to achieve maximum density values with minimum grain coarsening. Prepared samples were $15 \mathrm{~mm}$ diameter and $3 \mathrm{~mm}$ thickness, approximately.

\subsection{Density and microstructural characterization}

Bulk densities were measured by the Archimedes method using distilled water as immersion medium. Theoretical density values for the composites were calculated by the rule of mixtures assuming density values of $3.97 \mathrm{~g} \mathrm{~cm}^{-3}$ for $\mathrm{Al}_{2} \mathrm{O}_{3}$ and $1.80 \mathrm{~g} \mathrm{~cm}^{-3}$ for SWNTs.

The structural integrity of SWNTs in the composites after SPS sintering was assessed by Raman spectroscopy performed on fracture surfaces using a dispersive microscope (Horiba Jobin Yvon LabRam HR800, Kyoto, Japan) equipped with a 20-mW He-Ne green laser $(532.14 \mathrm{~nm})$.

Microstructural studies of composite fracture surfaces have been performed by highresolution scanning electron microscopy HRSEM (HITACHI S5200) to analyze the distribution of SWNTs in the alumina matrix, and to characterize the ceramic grains morphology. Distribution and morphology of SWNT agglomerates present in the composites were characterized by low magnification conventional SEM (JEOL 6460LV). Cross-section (c.s.) and in-plane (i.p.) slices, i.e. surfaces parallel and perpendicular to the SPS pressing direction were polished with diamond paste up to 1 $\mu \mathrm{m}$ for morphological studies. Additionally, polished surfaces devoted to characterize the alumina grains were thermally etched at $1200{ }^{\circ} \mathrm{C}$ for 20 minutes in air to reveal grain boundaries. The morphology characterization was made measuring about 200 grains or 
agglomerates respectively to obtain the equivalent planar diameter as size parameter, $\mathrm{d}$ $($ or $\mathrm{D})=2(\text { area } / \pi)^{1 / 2}$, and the shape factor, $\mathrm{f}($ or $\mathrm{F})=(4 \pi \cdot$ area $) /(\text { perimeter })^{2}$. Hereafter, lowercase letters will refer to alumina parameters and uppercase letters to agglomerates. Surface density of agglomerates was evaluated from the area fraction covered by them in low magnification SEM micrographs.

\subsection{Mechanical testing}

Vickers hardness measurements were carried out on samples polished up to $1 \mu \mathrm{m}$ diamond paste. 19.6 N load was applied for 10 seconds using a diamond Vickers indenter (Duramin Struers, Germany). Vickers hardness tests were performed on both c.s. and i.p. surfaces. 30 indents were made on each surface avoiding boundary effects (i.e. keeping the appropriate distance from sample edges and between indentation marks), in well-separated and randomly selected regions to verify data consistency. Only 15 indents were performed on each orientation in monolithic alumina for comparison. The hardness value $\mathrm{H}_{\mathrm{V}}$ (in $\mathrm{GPa}$ ) was calculated from the indentation load $\mathrm{P}$ and the diagonal of the Vickers imprint $\mathrm{a}: \mathrm{H}_{\mathrm{V}}=1.854\left(\mathrm{P} / \mathrm{a}^{2}\right)$.

For samples exhibiting more than 9 suitable tests, the $\mathrm{H}_{\mathrm{V}}$ data-set was statistically analyzed using a 2-parameter Weibull distribution to quantify its scatter. According to Weibull statistics, the cumulative probability $(p)$ of a parameter, $H_{V}$ in our case, can be expressed as: $p=1-\exp \left[-\left(H_{V} / H_{V_{0}}\right)^{m}\right]$ where $H_{V O}$ is the Weibull scale parameter, i.e. the characteristic value of hardness having $63.2 \%$ probability, and $m$ is the Weibull modulus that describes the extent of scatter in a given data-set. The cumulative probability can be obtained from empirical data using the approach: $p_{i}=(i-0.5) / n$ where $i$ is the rank of the $i$ th observation corresponding to data-set arranged in ascending order and $\mathrm{n}$ is the total number of observations.

$H_{V 0}$ parameter is a measure of the nominal hardness of the material and usually increasing $H_{V O}$ values will be found with increasing values of average hardness. Parameter $m$ is related to the reliability of a distribution, so higher $m$ values indicate a lower scatter (narrower data distribution) and, hence, higher reliability. Thus a $1 /(1-\mathrm{p})$ 
plot on a natural $\log (\log )$ scale versus $H_{V} / H_{V_{0}}$ on a natural $\log$ scale should give a straight line of slope $m$ and from its intercept can be evaluated $H_{V_{0}}$ value.

\section{Results and discussion}

\subsection{Microstructural characterization}

Table 1 shows the density values of monolithic alumina and $\mathrm{SWNT} / \mathrm{Al}_{2} \mathrm{O}_{3}$ composites after consolidation by SPS. The nomenclature used to refer to the composites is also included. The increase in relative density of $\mathrm{SWNT} / \mathrm{Al}_{2} \mathrm{O}_{3}$ composites with rising carbon nanotubes content implies that SWNTs promote densification in these materials. Present results differ from most of the published results on $\mathrm{CNT} / \mathrm{Al}_{2} \mathrm{O}_{3}$ composites $[9,10,12,14,16]$ where CNTs inhibit densification of $\mathrm{Al}_{2} \mathrm{O}_{3}$. Only few works report relative densities $\sim 100 \%$ [8] or densification enhancement with CNT incorporation $[11,17]$. In our case, this favorable sinterability suggests the presence of an effective diffusion layer bonding the SWNTs and the alumina grains. According to the sintering temperature used, the formation of an aluminum oxy-carbide phase (Al-O-C interphase among SWNTs and $\mathrm{Al}_{2} \mathrm{O}_{3}$ grains) is possible [22,24].

Figure 1 presents the characteristic fracture surfaces exhibited by the composites which illustrate the adequate dispersion of SWNT bundles surrounding alumina grains. Transgranular fracture zones are appreciated in composite $\mathrm{C} 1$, while the other composites mostly exhibit intergranular fracture. CNTs remain attached to the grains taking their shape and no pull-out is observed after intergranular fracture. SWNTs located mainly parallel to the fracture surface, implying few pull-outs, have also been observed in SWNT reinforced zirconia toughened alumina (ZTA) composites [25]. Whereas the alumina grains surrounded by SWNTs are scarce in C1, it appears that SWNTs cover most of the grain boundaries in the composites with higher CNT content. The series of high-magnification micrographs show that SWNTs look like a black covering that coats an increasing portion of alumina grain surfaces with increasing SWNT content. The higher the amount of carbon nanotubes, the larger the grain surface coated by them. Dark regions are composed by SWNTs as confirmed by Raman spectroscopy (not shown). Similar microstructure has been previously observed in other 
SWNT/ $/ \mathrm{Al}_{2} \mathrm{O}_{3}$ composites $[6,19,20]$. In MWNT/ $/ \mathrm{Al}_{2} \mathrm{O}_{3}$ nanocomposites only Huang et al. [26] observed this particular microstructure also in their fracture surface. These authors relate the observation of printed CNTs like stamps on the alumina grains with carbon diffusion into alumina lattice [26]. This idea suggests that the appearance of CNTs like a blanket coating the grains observed in these composites could be indicative of the presence of $\mathrm{Al}-\mathrm{C}-\mathrm{O}$ interphase between $\mathrm{CNTs}$ and alumina grains referred by $[22,24]$.

Table 1 displays global results of morphological parameters of alumina grains. No differences were observed on results from i.p. and c.s. surfaces, so the mean grain size, its standard deviation and the shape factor values presented in table 1 are the average parameters from both orientations. HRSEM observations of polished and thermally etched surfaces evidenced the existence of anomalous alumina grain growth together with an elongated aspect of the matrix grains regardless the composition and orientation (not shown). Similar mean grain size (below $1 \mu \mathrm{m}$ ) and shape factor (about 0.7) were measured in composites with lower SWNT content (C1 and C3) and no significant differences were observed compared to monolithic alumina grains [23] except for narrower size distributions $\left(\right.$ smaller $\sigma_{\mathrm{d}}$ ). Conversely, finer grain size with narrower distributions were found in composites with high carbon nanotube content (C5 and $\mathrm{C} 10)$, but differences in relation to the grain shape were not appreciated.

Low-magnification SEM micrographs (figure 2) illustrate the arrangement and morphology of SWNT agglomerates in the i.p. and the c.s. surfaces. Although many processing efforts were devoted to obtain a homogeneous distribution of SWNTs, it is obvious that the presence of agglomerates in the composites has not been avoided. It is interesting to note that the existence of agglomerates has not entailed detrimental effect on density values (table 1). The rough finish observed is due to pull-out of the alumina grains, which takes place during the polishing process of the composites. This is in agreement with the observations of Echeberria et al. [25] on rougher finishing in surfaces with small alumina grains and CNTs compared to flat finishing in areas consisting of large-grains without CNTs. These latter zones are inexistent in our composites due to the adequate dispersion of SWNTs achieved.

While the agglomerates (dark phase) are randomly oriented on the i.p. surface (fig. 2a), a strong alignment is clearly observed from micrographs on the c.s. plane (fig. 2b), with 
their major axis disposed perpendicular to the direction of applied pressure during sintering. Only Thomson et al. [7] have also referred anisotropic orientation of CNT agglomerates in alumina. Similar agglomerate size, about $6-8 \mu \mathrm{m}$ (table 2), was found regardless the SWNT content on the i.p. sections. Smaller sizes were measured on the c.s. surfaces for each composite. Furthermore, agglomerate shapes were also very sensitive to orientation. Whereas more rounded shape is found on the i.p. surfaces $(\mathrm{F}=$ 0.6), a marked elongation was observed in the c.s. agglomerates $(F=0.4-0.5)$. These morphological characteristics suggest that the agglomerates are flattened structures.

Regarding the surface density of agglomerates $\rho_{\mathrm{s}}$, no remarkable differences have been found in relation to surface orientation. A considerable increase in the surface density of agglomerates with increasing SWNT content up to 5 vol. \% was observed, changing from $0.6 \%$ in $\mathrm{C} 1$ to $2.4 \%$ in $\mathrm{C} 5$ (table 3). However, similar agglomerate surface density was found for C5 and C10 composites. These results highlight the need to characterize agglomerate densities for each prepared composite with different SWNT content.

Assuming that the area fraction covered by agglomerates in SEM micrographs is equal to its volume fraction in composites (Delesse's principle of stereology), the percentage of the CNT content that are agglomerated was estimated from the bulk density of agglomerates and the SWNT content for each composition $\left(\mathrm{A} \%=100 \rho_{V} /\right.$ SWNT vol.\%). The SWNT vol. \% contained in the agglomerates can be directly inferred from agglomerate bulk density. The SWNT vol. \% dispersed at the grain boundaries is directly the difference between the total SWNT content and the SWNT content within the agglomerates. Hereinafter, A-SWNTs and GB-SWNTs will refer to SWNTs arranged in agglomerates and at grain boundaries respectively. From these calculations, similar percentages of the SWNT content $(\sim 40-60 \%)$ were agglomerated in composites up to 5 vol. \% SWNT, leading to both higher A- and GB-SWNT net contents in composites with higher SWNT content (table 3). This percentage is even smaller in the case of $\mathrm{C} 10(\sim 20 \%)$ than for the former compositions, which results in a net A-SWNT content similar to that of composite C5 and a larger amount of SWNTs distributed at the grain boundaries. Although it has generally been assumed by other authors that agglomeration increases in case of high CNT content due to dispersion 
difficulties, the results presented here point otherwise. These calculations are in agreement with increasing amounts of SWNT bundles embedded among ceramic grain boundaries observed by HRSEM (fig. 1). Besides, the matrix refinement found in high SWNT content composites (C5 and C10, table 1) also supports that the predominant volume fraction of SWNTs is placed in networks surrounding the ceramic grains. Despite the presence of agglomerates, the GB-SWNT fraction has effectively inhibited the matrix grain growth since the presence of SWNTs at ceramic grain boundaries disfavor both grain boundary sliding and diffusion during densification [27,28].

\subsection{Evaluation of Vickers hardness in $\mathrm{SWNT} / \mathrm{Al}_{2} \mathrm{O}_{3}$ composites}

Since microstructural characterization revealed anisotropy in agglomerate morphology, hardness both on i.p. and c.s. surfaces was evaluated. Regarding the formation of the indentation marks, most of the i.p. imprints were not well defined for all composites, i.e. the diagonals of the Vickers imprint were not visible (fig. 3a), while a higher number of proper well formed indentations were obtained on c.s. surfaces (fig. 3b). Up to our knowledge, no similar findings have been published in literature. Since Vickers hardness is calculated from diagonals of indenter imprints, the topography of each imprint was examined to discard those without the characteristic Vickers indenter regular shape. These optical images of typical indenter marks also show that polished surfaces have a rough finish due to grain pull-out during polishing. Vickers sites also exhibit typical lack of classical radial cracks in the indentation impression [6,25].

A trend of decreasing on the number of Vickers marks showing visible diagonals with increasing SWNT content can be clearly appreciated for all compositions in the bar chart graph of figure 4. Furthermore, these results also point out that the use of crosssection surface allows an accurate measurement of Vickers hardness in these composites more efficiently. As an example of the inefficiency of the procedure on i.p. surface, after 30 indentations performed in $\mathrm{C} 3$, only one proper measure was accomplished (fig. 4). In contrast, 24 proper imprints were obtained on c.s. surface for the same number of performed indentations. For composites with higher SWNT content, no suitable imprints were found on their respective i.p. surfaces (therefore, bars of $\mathrm{C} 5$ and $\mathrm{C} 10$ are not shown for these cases in fig. 4). 
Since the ceramic grain morphology resulted very similar in both i.p. and c.s. surfaces, any discrepancies found in the definition of the imprint between both surfaces may be due to a different cause, most likely related to the different arrangement and shape of the CNT agglomerates. Taking into account that the agglomerates dispersed in the alumina matrix are SWNT entangled networks, they are bent during loading acquiring the shape of the indenter. Once the load is removed they are expected to elastically spring back to their original conformation.

Therefore, SWNT aggregates can be visualized as elastic networks which prevent plastic deformation by indentation. Recently, Sarkar and Das [22] referred MWNTs stayed intact in their positions in indented regions of pressure-less sintered MWNT/alumina composites, while alumina matrix exhibited permanent deformation. The finding of unaffected carbon nanotubes by the indenter tip-CNT direct interaction during the indentation load cycle [22] supports that the contact between the indenter tip and elastic SWNT agglomerates prevents the formation of imprints properly. The probability of finding agglomerates is proportional to the agglomerate surface density. When the indenter is pushed in the direction of the sintering applied pressure, the rounded shape and larger size of the agglomerates favors greater contact surface with the indenter. However, in the cross-section areas the agglomerates exhibit smaller size with a much higher aspect ratio, very thin and elongated in the direction perpendicular to the applied pressure, leaving larger agglomerate-free areas in contact with the indenter, which allows permanent deformation of the matrix during indenter penetration. This idea could also explain the decrease in the number of suitable imprints with increasing SWNT content, which would be due to the increase of agglomerate density. This also suggests a control technique to compare SWNT dispersion in these composites consisting of making a fixed number of Vickers indentations so that the number of unsuitable marks can roughly provide direct information on the level of SWNT agglomeration. The decreasing tendency in the number of proper marks observed between $\mathrm{C} 5$ and $\mathrm{C} 10$ for indentations performed on their cross-section surfaces (figure 4) suggests that the extent of SWNT layer covering the alumina grains could also affects the imprint formation. These CNTs are randomly dispersed in the alumina matrix and acquire the tortuous shape of alumina grain boundaries. Our results suggest that the higher the surface contact among indenter and SWNTs (both in agglomerates as at grain boundaries), the greater the number of unsuitable indentations. 
Vickers hardness values were evaluated only from indentations showing well defined marks. Values determined for each material resulted very similar both for the different orientations examined and for the different scanned zones on each surface (table 4). Therefore, although applied pressure during SPS leads to preferential alignment of SWNT agglomerates perpendicular to the pressing direction, directly observed on agglomerates distribution (fig. 2b), anisotropic effects have not been observed in the values of Vickers hardness for these $\mathrm{SWNT} / \mathrm{Al}_{2} \mathrm{O}_{3}$ composites. Except for the composite $\mathrm{C} 1$ whose hardness is similar to that of monolithic alumina, $\mathrm{H}_{\mathrm{V}}$ values of the composites with higher SWNT content are lower. This can be explained by the fact that SWNTs are a softer component [17]. Despite the drop observed in composite hardness with respect to alumina, it is interesting to note the high hardness value measured for $\mathrm{C} 5$ on c.s. surface $(15.4 \mathrm{GPa})$, a value $40 \%$ higher than that found for a similar composite also indented on its c.s. surface [7].

The greater number of proper imprints found in c.s. surfaces allows a higher accuracy on the hardness measurements performed on these surfaces in comparison to those carried out on i.p. surfaces. This is supported by the reduction of the standard deviation of the data measured on c.s. surfaces due to the greater number of appropriate measurements (table 4). Therefore, the indentation of the cross-section surface is proposed for these composites in order to achieve superior preciseness in hardness measurement.

Weibull plots (figure 5) of $\mathrm{H}_{\mathrm{V}}$ data obtained from indentations performed on c.s. surfaces of the composites show satisfactory linear fits consistent with the Weibull distribution proposal. Results of Weibull statistics are tabulated in table 4.

A decreasing trend of the Weibull moduli (i.e. slope of the linear regression lines) with increasing SWNT content is observed. Hence, the $\mathrm{H}_{\mathrm{V}}$ data present a higher scatter in composites with higher SWNT content because the lower $m$ means the greater the variability of hardness. Compared to monolithic alumina $\left(m_{\mathrm{Al}_{2} \mathrm{O}_{3}}=40.2\right)$, the addition of SWNTs leads to a poorer structural reliability (i.e. low $m$ values). Notwithstanding the above, the decrease is not very pronounced in the case of low carbon nanotube content $\left(m_{C 1}=31.2\right)$. Nearly invariable reliability was found for C3 $\left(m_{C 3}=15.0\right)$ and C5 $\left(m_{C 5}=\right.$ 14.8) composites. Such large variability would lead to uncertainties in obtaining a 
precise $\mathrm{H}_{V}$ value for composites with high SWNT content. Since the agglomerate density for all these composites is different, this similarity in Weibull moduli found for $\mathrm{C} 3$ and $\mathrm{C} 5$ rules out that the increased scatter of $\mathrm{H}_{\mathrm{V}}$ data with increasing SWNT content from 1 to 3 vol. \% is due to a greater presence of agglomerates. The fact that interfacial bonding between alumina grains degrades with SWNT addition, in agreement with increasing tendency to intergranular fracture exhibited by the composites (fig. 1), suggests that GB-SWNTs could be the weakest flaw controlling the $\mathrm{H}_{\mathrm{v}}$ scatter. The decreasing trend of Weibull moduli with increasing SWNT content up to 3 vol. \% could be related to higher possibility of indentation zones with alumina grains surrounded by GB-SWNTs. HRSEM inspections support that the alumina grains surrounded by SWNTs were scarce in $\mathrm{C} 1$ and an increasing fraction of alumina grains was coated by carbon nanotubes with increasing SWNT content (fig. 1). Once the GB-SWNTs cover most of the grain boundaries in the composites, this effect saturates leading to a stabilization of $m$ value in composites with higher CNT content (C3 and C5). Thus, the continuity degree in the GB-SWNT distribution could be the key point controlling the variability of hardness.

The characteristic values of Weibull distributions $H_{V O}$ exhibit the similar decreasing trend than transversal $\mathrm{H}_{\mathrm{V}}$ data with increasing SWNT content (i.e., leftward shift observed in Weibull plots for composites), changing from 19.3 to $15.9 \mathrm{GPa}$ for composites with 1 and 5 vol. \% SWNT, respectively. The $\mathrm{C} 1$ composite shows a slight increase in $H_{V O}$ compared with the value of $19.0 \mathrm{GPa}$ found for monolithic alumina in agreement to mean $\mathrm{H}_{\mathrm{V}}$ values also reported in table 4. The composites $\mathrm{C} 1$ and $\mathrm{C} 3$ exhibited similar matrix microstructures whereas C5 even showed refined grain size (table 1). Therefore, the $\mathrm{H}_{\mathrm{V} 0}$ decreasing trend with increasing SWNT content observed in figure 5 is not expected from matrix microstructure differences. Moreover, the increasing agglomerate surface densities found in these composites lead to similar percentages of SWNT content arranged into agglomerates in all them with increasing both A- and GB-SWNT vol. \% as SWNT content increases (table 3). The hardness decrease could be explained arguing that CNT incorporation involves the addition of a softer phase and interfacial bonding between alumina grains worsens with SWNT addition. Therefore, more quantity of SWNTs at grain boundaries must be the responsible of the $H_{V O}$ decrease. 
Performing similar statistical analysis, an improvement of $\mathrm{H}_{\mathrm{V}}$ in pressure-less sintered MWNT/alumina composites has been reported by Sarkar and Das [22]. These authors propose that the adequate grain boundary cohesion achieved by flexible multi-walled carbon nanotubes entangling alumina grains could be responsible of the superior $\mathrm{H}_{\mathrm{V}}$ reliability of the composites over pure alumina despite the presence of CNT agglomerates. Our results point in the opposite direction to that suggested by the previous authors. SWNT addition reduces the reliability of hardness values in aluminabased composites. It affects negatively the grain boundary cohesion in SWNT/alumina composites and SWNTs distributed at grain boundaries are pointed as the critical flaw. It is suggested that the fraction of alumina grains surrounded by SWNTs controls the variability of hardness values, whereas the increasing amount of GB-SWNTs is responsible of the decrease in characteristic hardness values with increasing SWNT content. The presence of agglomerates does not play a fundamental role on the $\mathrm{H}_{\mathrm{V}}$ distribution in these composites.

\section{Conclusions}

$\mathrm{SWNT} / \mathrm{Al}_{2} \mathrm{O}_{3}$ composites with carbon nanotube content from 1 to $10 \mathrm{vol} \%$ were prepared by colloidal processing and SPS. An increasing trend in their relative density with increasing SWNT content has been observed. Dense composites with submicron alumina matrix were obtained with SWNTs distributed at grain boundaries and into agglomerates homogeneously dispersed. The agglomerate size and shape resulted very sensitive to orientation, exhibiting a much higher elongation in the direction perpendicular to the applied pressure in cross-section areas than on in-plane surfaces where agglomerates were more rounded without exhibiting preferred orientation. The characterization of SWNT agglomerates performed in this work indicates that increasing SWNT content (from 1 to 5 vol. \%) involves similar percentage of the SWNT content placed in agglomerates $(\sim 50 \%)$.

A decreasing trend of the number of Vickers marks showing visible diagonals with increasing SWNT content was clearly appreciated for all compositions. In order to optimize the performance of Vickers hardness tests, indentation on the cross-section surfaces is proposed. Superior hardness measurement reliability when testing on crosssection surfaces over in-plane ones is mainly attributed to the different agglomerate 
morphology observed between these orientations. A control technique to compare SWNT level of agglomeration in these composites is proposed consisting of evaluating the number of imprints without pyramidal diagonal marks after making a fixed number of Vickers indentations. This number provides rough information on the level of SWNT agglomeration.

Although applied pressure during SPS leads to preferential alignment of SWNT agglomerates perpendicular to the pressing direction, anisotropic effects have not been observed in the values of Vickers hardness. The decreasing trend in $\mathrm{H}_{\mathrm{V}}$ with increasing SWNT content observed for these $\mathrm{SWNT} / \mathrm{Al}_{2} \mathrm{O}_{3}$ composites has been explained by the presence of higher SWNT quantities at grain boundaries. The presence of agglomerates does not play a fundamental role on the $\mathrm{H}_{\mathrm{V}}$ distribution in these composites.

\section{Acknowledgements}

The authors would like to acknowledge the financial support obtained from the Spanish Ministry of Science and Innovation (MAT2009-11078 and MAT2012-34217) and from Junta de Andalucía (P12-FQM-1079). Microscopy studies have been performed in facilities belonging to the CITIUS (Universidad de Sevilla). 


\section{References}

[1] Ijima S, Brabec CH, Maiti A, Bernholc ZJ. Structural flexibility of carbon nanotubes. Carbon 1995; 33: 925-30.

[2] Treacy MM, Ebbesen TW, Gibson JM. Exceptionally high Young's modulus observed for individual carbon nanotubes. Nature 1996; 381: 678-80.

[3] Subramoney S. Nanocarbons-structure, properties, and potential applications. Adv Mater 1998; 15: 1157-71.

[4] Yu MF, Files BS, Arepalli S, Ruoff RS. Tensile loading of ropes of single wall carbon nanotubes and their mechanical properties. Phys Rev Lett 2000; 84: 5552-55.

[5] Yamamoto G, Omori M, Yokomizo K, Hashida T. Mechanical properties and structural characterization of carbon nanotube/alumina composites prepared by precursor method. Diamond Relat Mater 2008; 17: 1554-57.

[6] Wang X, Padture NP, Tanaka H. Contact-damage-resistant ceramic/single-wall carbon nanotubes and ceramic/graphite composites. Nat Mater 2004; 3: 539-44.

[7] Thomson KE, Jiang D, Yao W, Ritchie RO, Mukherjee AK. Characterization and mechanical testing of alumina-based nanocomposites reinforced with niobium and/or carbon nanotubes fabricated by spark plasma sintering. Acta Mater 2012; 60: 622-32.

[8] Zhan GD, Kuntz JD, Wan J, Mukherjee AK. Single-wall carbon nanotubes as attractive toughening agents in alumina-based composites. Nat Mater 2003; 2: 38-42.

[9] Fan JP, Zhuang DM, Zhao DQ, Wu MS, Wei F, Fan ZJ. Toughening and reinforcing alumina matrix composite with single-wall carbon nanotubes. Appl Phys Lett 2006; 89: 121910.

[10] Fu ZY, Huang LW, Zhang JY, Todd R. Ultra-fast densification of CNTs reinforced alumina based on combustion reaction and quick pressing. Sci China Tech Sci 2012; 55: 484-9.

[11] Mo CB, Cha SI, Kim KT, Lee KH, Hong SH. Fabrication of carbon nanotube reinforced alumina matrix nanocomposite by sol-gel process. Mater Sci Eng A 2005; 395: 124-8.

[12] Zhang T, Kumari L, Du GH, Li WZ, Wang QW, Balani K, Agarwal A. Mechanical properties of carbon nanotube-alumina composites synthesized by chemical vapor deposition and spark plasma sintering. Composites: Part A 2009; 40: 86-93.

[13] Lee K, Mo CB, Park SB, Hong SH. Mechanical and electrical properties of multiwalled CNT-alumina nanocomposites prepared by a sequential two-step 
processing of ultrasonic spray pyrolysis and spark plasma sintering. J Am Ceram Soc 2011; 94: 3774-9.

[14] Bakhsh N, Khalid FA, Hakeem AS. Synthesis and characterization of pressureless sintered carbon nanotube reinforced alumina nanocomposites. Mater Sci Eng A 2013; 578: 422-9.

[15] Yamamoto G, Omori M, Hashida T, Kimura H. A novel structure for carbon nanotube reinforced alumina composites with improved mechanical properties. Nanotech 2008; 19: 315708.

[16] S. Sarkar, P. Kr. Das. Microstructure and physicomechanical properties of pressureless sintered multiwalled carbon nanotube/alumina nanocomposites. Ceram Int 2012; 38: 423-32.

[17] Zhang J, Xu C, Todd R, Fu Z. Vickers hardness of multi-wall carbon nanotube reinforced alumina. Adv Mater Res 2013; 750-752: 541-5.

[18] Zapata-Solvas E, Gómez-García D, Domínguez-Rodríguez A. Towards physical properties tailoring of carbon nanotubes-reinforced ceramic matrix composites. J Eur Ceram Soc 2012; 32: 3001-20.

[19] Poyato R, Vasiliev AL, Padture NP, Tanaka H, Nishimura T. Aqueous colloidal processing of single-wall carbon nanotubes and their composites with ceramics. Nanotech 2006; 17: 1770-7.

[20] Vasiliev AL, Poyato R, Padture N. Single-wall carbon nanotubes at ceramic grain boundaries. Scripta Mater 2007; 56: 461-3.

[21] Poorteman M, Traianidis M, Bister G, Cambier F. Colloidal processing, hot pressing and characterisation of electroconductive MWCNT-alumina composites with compositions near the percolation threshold. J Eur Ceram Soc 2009; 29: 669-75.

[22] Sarkar S, Das PK. Statistical analysis of mechanical properties of pressureless sintered multiwalled carbon nanotube/alumina nanocomposites. Mater Chem Phys 2012; 137: 511-8.

[23] Morales-Rodríguez A, Poyato R, Gallardo-López A, Muñoz A, DomínguezRodríguez A. Evidence of nanograin cluster coalescence in spark plasma sintered $\alpha-\mathrm{Al}_{2} \mathrm{O}_{3}$. Scripta Mater 2013; 69: 529-32.

[24] Ahmad I, Unwin M, Cao H, Chen H, Zhao H, Kennedy A, Zhu YQ. Multi-walled carbon nanotubes reinforced $\mathrm{Al}_{2} \mathrm{O}_{3}$ composites: Mechanical properties and interfacial investigations. Comp Sci Tech 2010; 70: 1199-206. 

Reyes A, Domínguez-Ríos C, Aguilar-Elguézabal A, Bocanegra-Bernal MH. Hard and tough carbon nanotube-reinforced zirconia-toughened alumina composites prepared by spark plasma sintering. Carbon 2012; 50: 706-17.

[26] Huang Q, Jiang D, Ovid'ko IA, Mukherjee AK. High-current-induced damage on carbon nanotubes: The case during spark plasma sintering. Scripta Mater 2010; 63: $1181-4$.

[27] Zapata-Solvas E, Poyato R, Gómez-García D, Domínguez-Rodríguez A, Radmilovic V, Padture NP. Creep-resistant composites of alumina and single-wall carbon nanotubes. Appl Phys Lett 2008; 92: 111912.

[28] Inam F, Yan H, Peijs T, Reece MJ. The sintering and grain growth behaviour of ceramic-carbon nanotube nanocomposite. Comp Sci Tech 2010; 70: 947-52. 


\section{Figure captions}

Figure 1. HRSEM micrographs of fracture surfaces: (a) C1, (c) C3, (e) C5 and (g) C10. Details corresponding to higher-magnification micrographs are shown in (b), (d), (f) and (h). The dark regions are SWNT bundles (arrows) surrounding alumina grains (light regions).

Figure 2. SEM micrographs showing the SWNT agglomerate distribution on (a) inplane and (b) cross-section surfaces of composite C10. Compression axis during SPS is indicated in (b) by arrows.

Figure 3. Optical images illustrating the different appearance of the imprints in $\mathrm{C} 1$ composite after indenting on (a) i.p. and (b) c.s. surfaces.

Figure 4. Statistical bar chart with percentages of proper indentations carried out on the different composites in both the in-plane (solid bars) and cross-section (hollow bars) surfaces.

Figure 5. Weibull plots of $\mathrm{H}_{\mathrm{V}}$ data of $\mathrm{C} 1, \mathrm{C} 3$ and $\mathrm{C} 5$ obtained on transversal surface orientation (solid regression lines). Data of monolithic $\mathrm{Al}_{2} \mathrm{O}_{3}$ are represented for comparison (dashed regression line). 
Table 1. Theoretical and relative density and morphological parameters of alumina grains.

\begin{tabular}{ccccccc}
\hline Material & $\begin{array}{c}\text { SWNT content } \\
(\text { vol. \% })\end{array}$ & $\begin{array}{c}\rho_{\mathrm{th}} \\
\left(\mathrm{g} \mathrm{cm}^{-3}\right)\end{array}$ & $\begin{array}{c}\rho_{\mathrm{r}} \\
(\%)\end{array}$ & $\begin{array}{c}<\mathrm{d}> \\
(\mu \mathrm{m})\end{array}$ & $\begin{array}{c}\sigma_{<\mathrm{d}>} \\
(\mu \mathrm{m})\end{array}$ & $\mathrm{f}$ \\
\hline $\mathrm{Al}_{2} \mathrm{O}_{3}{ }^{*}$ & 0 & 3.97 & 97.7 & 0.7 & 0.6 & $0.67 \pm 0.14$ \\
$\mathrm{C} 1$ & 1 & 3.95 & 98.5 & 0.6 & 0.3 & $0.68 \pm 0.10$ \\
$\mathrm{C} 3$ & 3 & 3.90 & 99.7 & 0.7 & 0.4 & $0.67 \pm 0.12$ \\
$\mathrm{C} 5$ & 5 & 3.87 & 99.8 & 0.5 & 0.4 & $0.71 \pm 0.12$ \\
$\mathrm{C} 10$ & 10 & 3.75 & 100 & 0.5 & 0.3 & $0.67 \pm 0.12$ \\
\hline
\end{tabular}

* Data from reference [23]. 
Table 2. Morphological parameters of SWNT agglomerates measured on i.p. and c.s. surfaces for each composite.

\begin{tabular}{|c|c|c|c|c|c|c|}
\hline \multirow[b]{2}{*}{ Material } & \multicolumn{3}{|c|}{ i.p. } & \multicolumn{3}{|c|}{ c.s. } \\
\hline & $\begin{array}{l}<D> \\
(\mu \mathrm{m})\end{array}$ & $\begin{array}{l}\sigma<\mathrm{D}> \\
(\mu \mathrm{m})\end{array}$ & $\begin{array}{c}F \\
( \pm 0.20)\end{array}$ & $\begin{array}{l}<D> \\
(\mu \mathrm{m})\end{array}$ & $\begin{array}{l}\sigma<\mathrm{D}> \\
(\mu \mathrm{m})\end{array}$ & $\begin{array}{c}F \\
( \pm 0.20)\end{array}$ \\
\hline $\mathrm{C} 1$ & 7.9 & 6.3 & 0.63 & 2.7 & 1.9 & 0.50 \\
\hline $\mathrm{C} 3$ & 6.4 & 3.8 & 0.61 & 5.5 & 3.9 & 0.53 \\
\hline C5 & 8.5 & 7.0 & 0.60 & 5.3 & 3.9 & 0.41 \\
\hline $\mathrm{C} 10$ & 7.6 & 5.7 & 0.56 & 6.0 & 6.0 & 0.43 \\
\hline
\end{tabular}


Table 3. Surface density of SWNT agglomerates, percentage of carbon nanotube content in agglomerates and A- and GB-SWNT vol. \% calculated for each composite.

\begin{tabular}{ccccc}
\hline Material & $\rho_{\mathrm{s}}(\%)$ & A\% & $\begin{array}{c}\text { A-SWNT } \\
\text { (vol. \%) }\end{array}$ & $\begin{array}{c}\text { GB-SWNT } \\
\text { (vol. \%) }\end{array}$ \\
\hline C1 & 0.6 & 60 & 0.6 & 0.4 \\
C3 & 1.3 & 43 & 1.3 & 1.7 \\
C5 & 2.4 & 48 & 2.4 & 2.6 \\
C10 & 2.1 & 21 & 2.1 & 7.9 \\
\hline
\end{tabular}


Table 4. Vickers hardness values of monolithic alumina and $\mathrm{SWNT} / \mathrm{Al}_{2} \mathrm{O}_{3}$ composites. The number of suitable measurements is indicated in parenthesis. Results of Weibull statistics for c.s. $\mathrm{H}_{\mathrm{V}}$ data are also tabulated.

\begin{tabular}{cccccc}
\hline \multirow{2}{*}{ Material } & \multicolumn{2}{c}{$\mathrm{H}_{\mathrm{V}}(\mathrm{GPa})$} & \multicolumn{2}{c}{ Weibull parameters } \\
\cline { 2 - 6 } & i.p. & c.s. & global & $m$ & $H_{V 0}(\mathrm{GPa})$ \\
\hline $\mathrm{Al}_{2} \mathrm{O}_{3}$ & $18.3 \pm 0.4(15)$ & $18.7 \pm 0.5(15)$ & $18.5 \pm 0.5(30)$ & $40.2 \pm 1.7$ & 19.0 \\
$\mathrm{C} 1$ & $18.7 \pm 1.5(8)$ & $18.9 \pm 0.7(27)$ & $18.9 \pm 1.0(35)$ & $31.2 \pm 1.5$ & 19.3 \\
$\mathrm{C} 3$ & $17.1(1)$ & $16.8 \pm 1.3(24)$ & $16.8 \pm 1.0(25)$ & $15.0 \pm 0.4$ & 17.3 \\
$\mathrm{C} 5$ & $\mathrm{n} / \mathrm{a}$ & $15.4 \pm 1.5(9)$ & $15.4 \pm 1.5(9)$ & $14.8 \pm 1.0$ & 15.9 \\
$\mathrm{C} 10$ & $\mathrm{n} / \mathrm{a}$ & $14.0 \pm 0.7(5)$ & $14.0 \pm 0.7(5)$ & n/a & n/a \\
\hline
\end{tabular}


Click here to download high resolution image
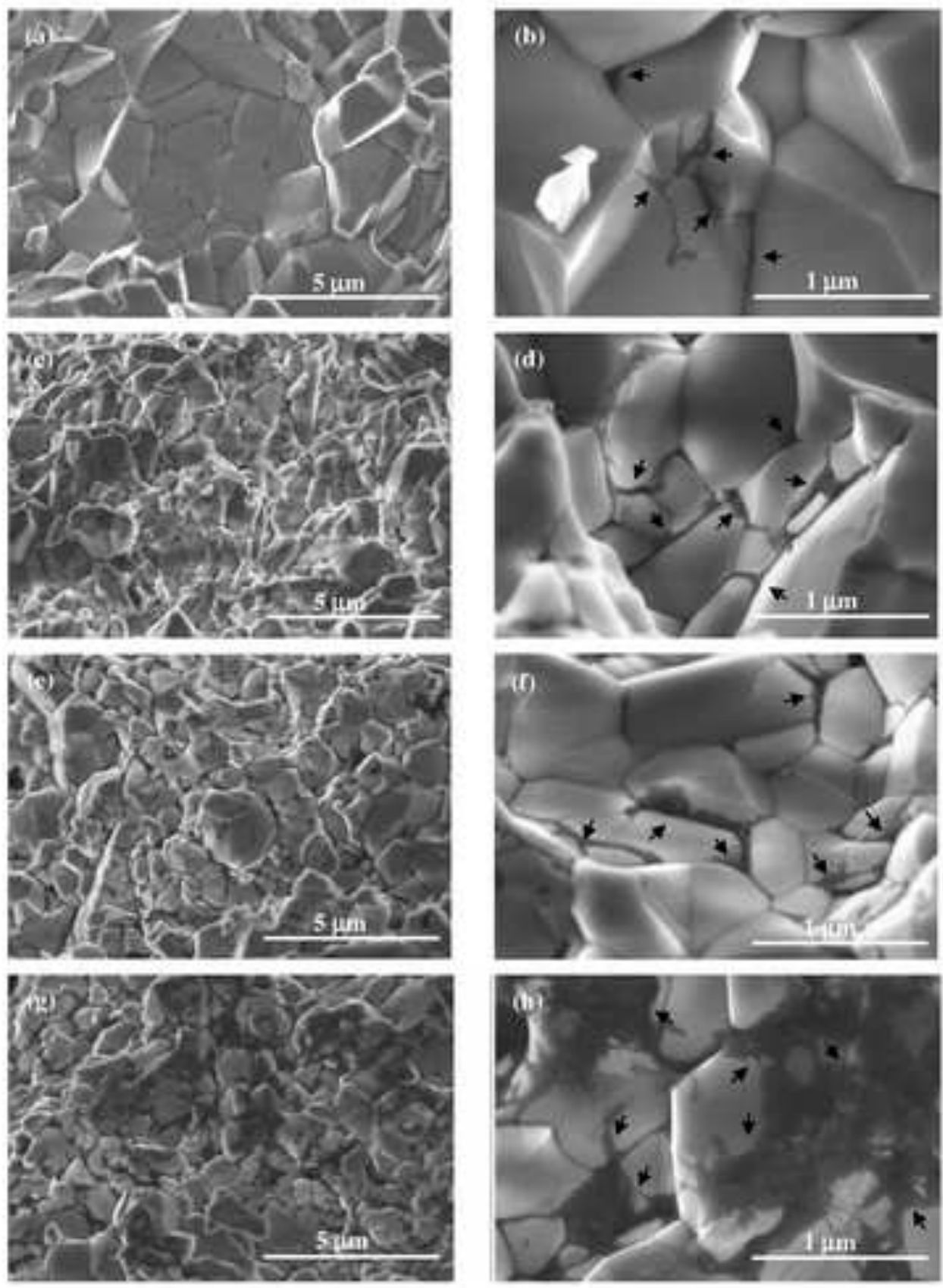
Click here to download high resolution image

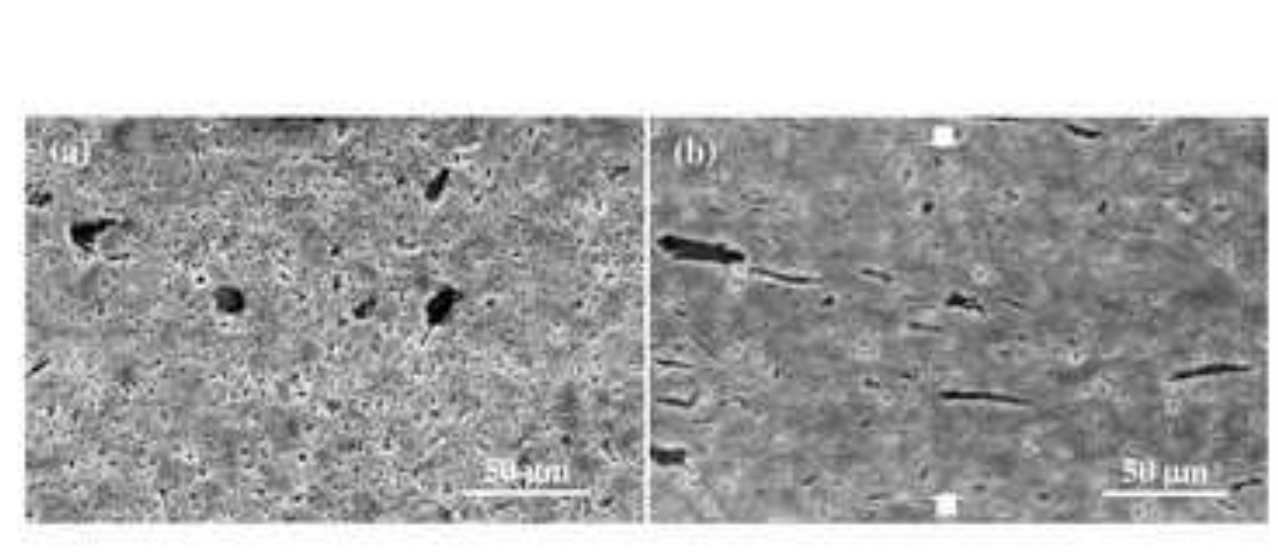

Figure

\author{
(
}

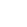

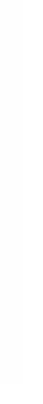


Click here to download high resolution image

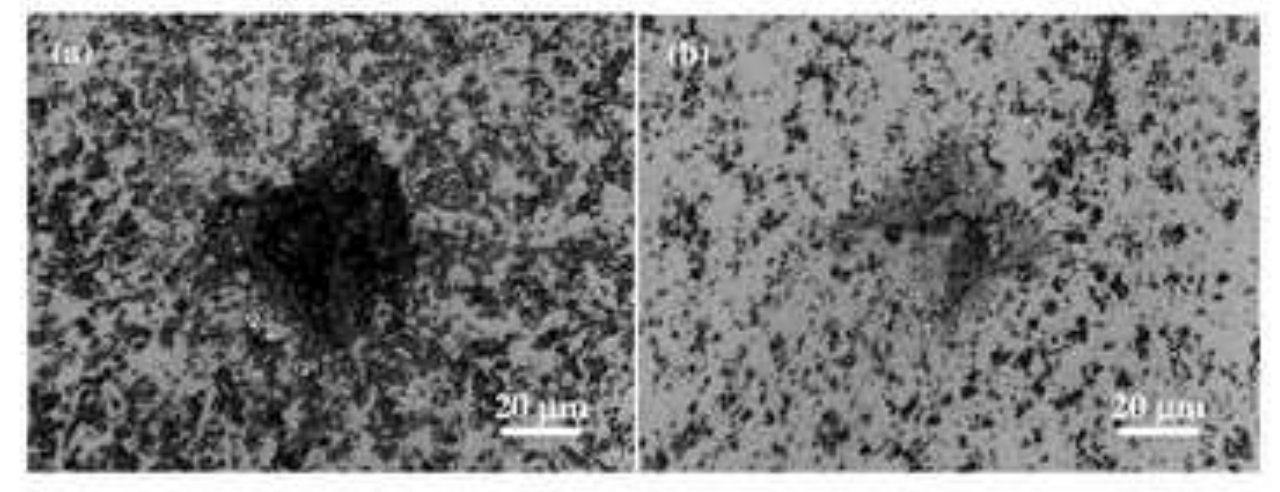

. 
Figure
Click here to download high resolution image

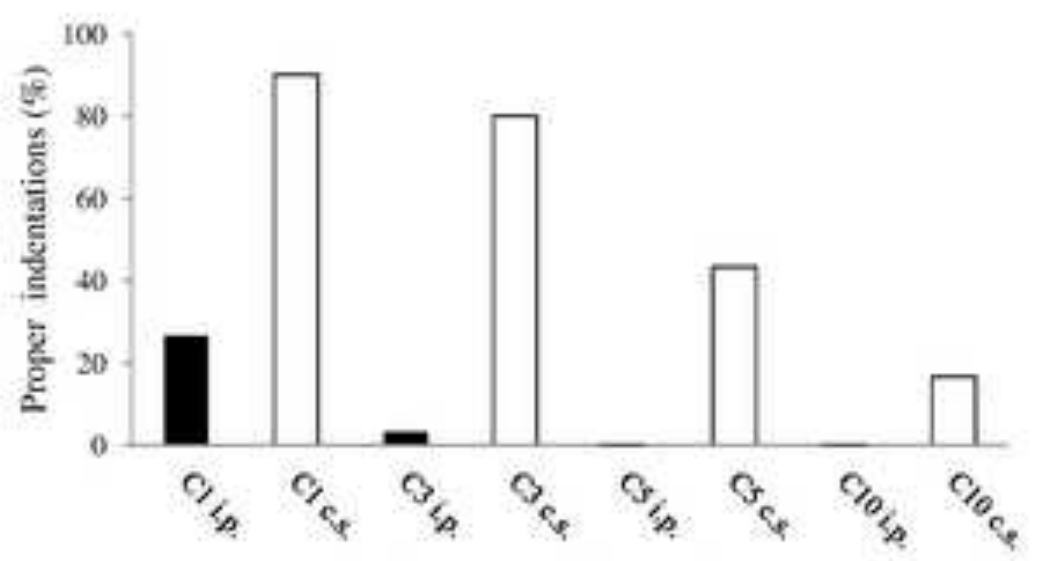

Composite and orientation 


\section{Figure}

Click here to download high resolution image

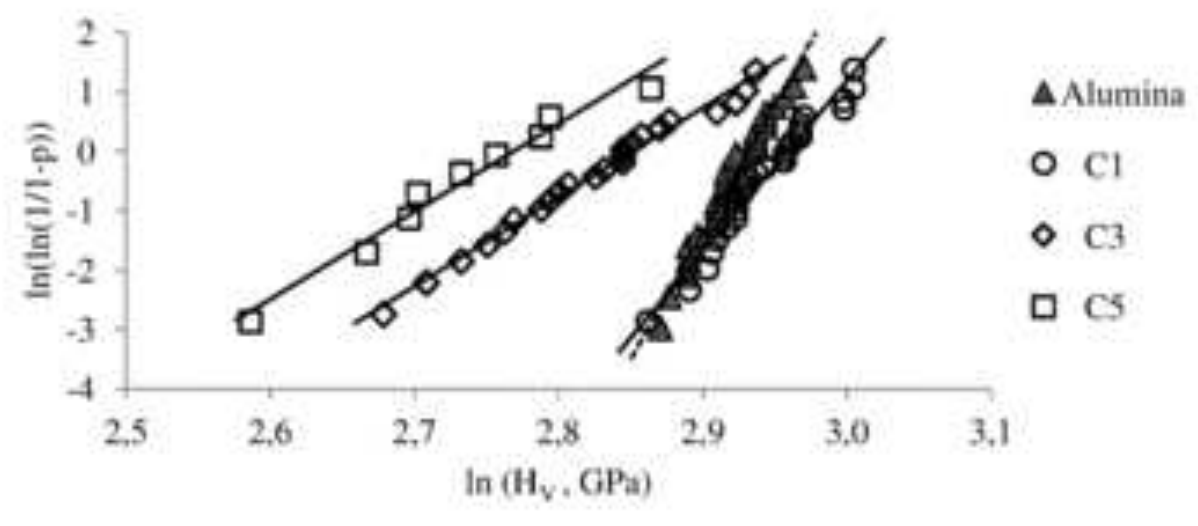


Figure 1. HRSEM micrographs of fracture surfaces: (a) C1, (c) C3, (e) C5 and (g) C10. Details corresponding to higher-magnification micrographs are shown in (b), (d), (f) and (h). The dark regions are SWNT bundles (arrows) surrounding alumina grains (light regions).

Figure 2. SEM micrographs showing the SWNT agglomerate distribution on (a) inplane and (b) cross-section surfaces of composite C10. Compression axis during SPS is indicated in (b) by arrows.

Figure 3. Optical images illustrating the different appearance of the imprints in $\mathrm{C} 1$ composite after indenting on (a) i.p. and (b) c.s. surfaces.

Figure 4. Statistical bar chart with percentages of proper indentations carried out on the different composites in both the in-plane (solid bars) and cross-section (hollow bars) surfaces.

Figure 5. Weibull plots of $\mathrm{H}_{\mathrm{V}}$ data of $\mathrm{C} 1, \mathrm{C} 3$ and $\mathrm{C} 5$ obtained on transversal surface orientation (solid regression lines). Data of monolithic $\mathrm{Al}_{2} \mathrm{O}_{3}$ are represented for comparison (dashed regression line). 